\title{
Effect of axial surface scores on rapid crack propagation in polyethylene pipe
}

\author{
L. Moreno ${ }^{1}$ and P. S. Leevers ${ }^{22}$
}

\begin{abstract}
Above a critical pressure and below a critical temperature, an internally pressurised plastic pipe can fail by sustained, axial, Rapid Crack Propagation (RCP). The fracture resistance of ductile pipe-grade polyethylenes is greater under plane stress than under plane strain - a fact which has important implications for large, thick-walled pipes. Previous small scale ISO 13477 'S4' tests on pipe have shown that a sharp, axial score on the inside pipe surface can suppress plane stress deformation during RCP, significantly reducing the critical pressure and increasing the critical temperature. This paper reports further results from S4 tests on specimens with sharp internal and external notches of controlled depth. The results are discussed in terms of ability of surface scores and notches to attract and guide cracks under different modes of pipe wall deformation, as well as their effect of reducing the resistance of the material to them.
\end{abstract}

\section{Introduction}

It has been known for plastic pipe since the work of Shannon and Wells ${ }^{1}$ and Greig et al. ${ }^{2}-$ and for steel pipe for much longer - that a gas-pressurised pipeline can fail by sustained, axial, rapid crack propagation (RCP). The speed of fracture, which may exceed that of sound in the pressurising fluid, is relevant to the mechanism by which the fluid transmits crack driving force through the structure. The crack speed is also a factor in reducing the fracture resistance of the pipe wall material: RCP in polyethylene leaves brittle, almost glassy fracture surfaces over most of which the normal bulk ductility of the material appears to have been suppressed. In thermoplastics, the loss of fracture resistance with increasing crack speed has been partly attributed (e.g. by Vincent ${ }^{3}$ ) to the increase in yield stress with strain rate. However, more recent work $^{4}$ suggests that the low resistance of thermoplastics to fast cracks results from the same ability to form a cracktip craze which gives them resistance to slow cracks. At high speed, the craze melts by adiabatic self-heating (a phenomenon whose importance at bulk scale Vincent had already recognised). The fracture resistance of pipe-grade polyethylene (PE) at high crack speeds can be as low as $2-4 \mathrm{~kJ} \mathrm{~m}^{-2}$. At low speeds, the fracture resistance is at least an order of magnitude greater, and crack propagation effectively becomes impossible.

The fracture resistance of $\mathrm{PE}$ is also much greater under plane stress conditions. A typical fracture surface

${ }^{1}$ Department of Material Sciences and Engineering, University of Toronto, Toronto, M5S 3E4, Canada

${ }^{2}$ Department of Mechanical Engineering, Imperial College London, London SW7 2AZ, UK

*Corresponding author, email p.leevers@imperial.ac.uk from a pipe wall more than $10 \mathrm{~mm}$ thick shows flat, brittle fracture over the central few millimetres, and progressively greater stress-whitening towards the margin of each free surface (Fig. 1). At these margins, tough PE grades may show gross ductility with $45^{\circ}$ shear separation, and the plane stress zone may be millimetres wide. For reasons which certainly include processed-in gradients of material properties through the pipe wall, the inside surface usually generates a considerably larger plane stress region, as Fig. 1 suggests. The contribution of the plane stress margins to the fracture resistance is particularly significant for pipe grade PE resins of the highest strength class, PE100. Its disproportionate contribution may offer hostages to fortune, one of which is investigated in the present paper.

Most of the observations described above were made using the small scale steady state ('S4') RCP test method for pipe, standardised as ISO 13477. Although it is now used primarily as a specifying test for commercial plastic pipe, the S4 method (shown schematically in Fig. 2) was originally developed as a research tool for the study of RCP in itself. RCP is initiated by radial, external impact of a sharp, chisel-bladed striker, but the initiation process is regarded as of only secondary importance. Impact resistance and RCP resistance are regarded as different properties, and this is not an impact test. Initiation takes place in a region which is, as far as possible, both pneumatically and mechanically isolated from the 'gauge region'. Initiation of a one-diameter arrest crack length at zero internal gauge pressure is sufficient to define valid test conditions, and the S4 method effectively designates the initiation zone as a 'free-fire zone' in which almost any means to achieve this criterion are permitted.

The gauge region of the test specimen is the propagation zone, in which both pressure and temperature are controlled. At test pressures above a well-defined

(C) 2004 loM Communications Ltd

Published by Maney for the Institute of Materials, Minerals and Mining

Received 15 January 2004; accepted 26 May 2004

Received 15 January 2004; accepte
DOI 10.1179/146580104225020983

Plastics, Rubbers and Composites

2004

VOL $33 \quad$ NO 4 

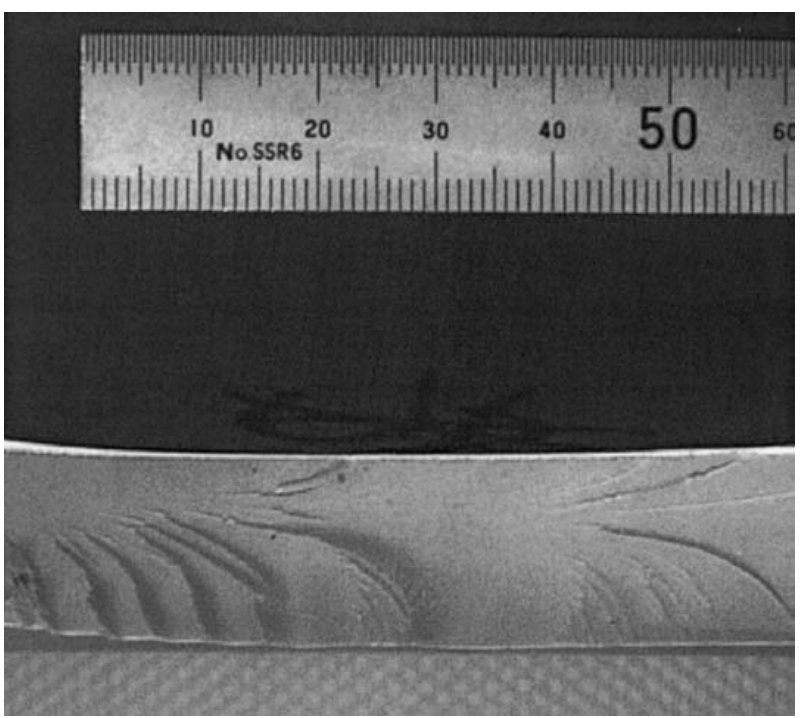

1 Fracture surface from RCP in a PE pipe, showing plane stress regions at inside (upper) and outside (lower) verges of crack path

critical value $p_{\mathrm{c}}, \mathrm{RCP}$ settles to a steady state within this zone and usually reaches the end of it. Below $p_{\mathrm{c}}$, the initiated crack continues to arrest promptly on entering it, at a length of around one pipe diameter $D$ from the impact point. ISO 13477 defines an arrest crack length of $4.7 \mathrm{D}$ as an RCP arrest/propagation criterion. To qualify pipe, $p_{\mathrm{c}}$ is determined from a series of $\mathrm{S} 4$ tests at constant temperature and different pressures.

During pioneering research using the S4 method, Yayla $^{6}$ discovered that a sharp, relatively shallow (1.5$2.0 \mathrm{~mm}$ ) razor notch along the bore surface had a remarkable effect on critical pressure at $0^{\circ} \mathrm{C}$. It is, of course, hardly surprising that locally reducing the pipe wall thickness from $B$ to $B_{\mathrm{c}}$ reduces the applied force needed to fracture it along the thinner path. Linear elastic fracture mechanics predicts that fracture load will change by a factor of $\sqrt{B_{\mathrm{c}} / B}$, but the observed decrease in $p_{\mathrm{c}}$ was much greater: in one material, from 10 to 0.75 bar. Morgan ${ }^{7}$ investigated further by fixing the test pressure and testing at a range of temperatures. The propagation/arrest transition then appears at a critical temperature $T_{\mathrm{c}}$. Morgan found that the $T_{\mathrm{c}}$ of a PE100 material could, when notched, increase from below $10^{\circ} \mathrm{C}$ to above $40^{\circ} \mathrm{C}$. Because the material tested by Yayla had a $T_{\mathrm{c}}$ value just above $0^{\circ} \mathrm{C}$, its critical pressure was very sensitive to temperature at $0^{\circ} \mathrm{C}$ so that the effect of a shift in $T_{\mathrm{c}}$ on critical pressure was dramatic.

The fact that notching lifts $T_{\mathrm{c}}$ above $0^{\circ} \mathrm{C}$ for PE100 resins was exploited in ISO13477, which allows internal notching of the initiation zone (only) as a means of achieving the $1 D$ initiation condition. Otherwise, very high blade impact speed or initiation zone supercooling must be used, either of which carries a much higher risk of contaminating conditions in the propagation zone. In PE100, notching is almost always needed to achieve valid $\mathrm{S} 4$ test initiation at $0^{\circ} \mathrm{C}$, the test temperature specified by some referring standards.

The S4 critical temperature phenomenon is in some ways analogous to the brittle-tough transition seen in impact tests, at which the brittle crack initiated under impact re-arrests before fracture. It has long been known that impact bend specimens of some tough

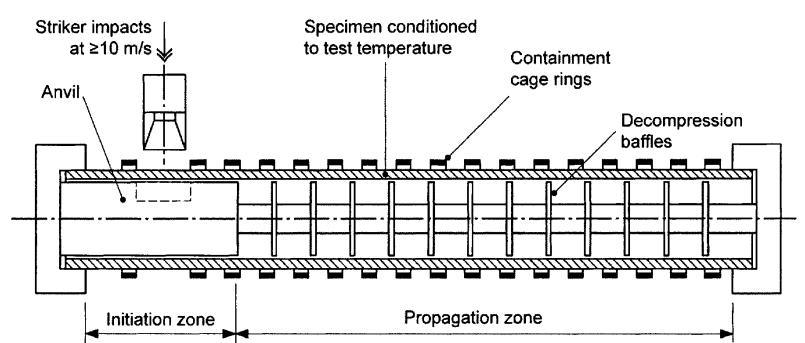

2 ISO 13477 'S4' pipe test

polymers were sensitive to sharp side-grooves. ${ }^{8}$ In this geometry, the effect on impact fracture resistance $G_{\mathrm{c}}$ is less than that on the brittle-tough transition temperature. However, in polyethylene, at least the transition temperature is not associated with an increase in $G_{\mathrm{c}}$, suggesting that the effect of a sharp side-groove must be exerted through changing resistance to rapid crack propagation rather than to impact fracture initiation. Since the stress intensity factor owing to a surface notch 1-3 mm deep is negligible compared to that at the through-thickness front of an extending crack, the notch effect is almost certainly not one of stress intensity superposition but rather one of constraint. If it is deep enough, the side-groove removes the crack path edge material which, under low constraint, could strongly resist fracture. If fine enough, however, each side-groove leaves enough material beside the new path edge so that it cannot 'dimple' inwards to relieve the triaxial constraint on it.

Because the S4 method is referenced from specifications for fuel gas and water distribution pipe, polymer suppliers have developed materials which perform very well in it. Nevertheless, the effect of surface notching on critical pressure - and the use of surface notching as an initiation strategy - have disturbed some pipe users and producers who remain wary of the S4 test. The method achieves a meaningful result on a small scale by suppressing the axial decompression which accompanies RCP at full scale. As a result, it inevitably sustains RCP at lower critical pressures, which must be scaled up using a correlation factor. The reduction in critical pressure caused by surface scoring can therefore look more dramatic than it is.

On the other hand, gas and water distribution pipe is not always treated kindly during installation procedures, and it may later be exposed to street works. The 'sliplining' installation procedures used to rehabilitate existing, damaged pipe can be particularly hard on the surface of these rather soft materials. It is not uncommon to see, at installation sites, PE pipe surfaces marked by heavy scores or drag marks. RCP events in plastic pipe network installations have always been rare during proof testing and are now almost unknown; and they have virtually never occurred in service. It is important that this record is maintained without the need to handle pipe with unrealistic care.

This paper reports results, selected from a long programme of S4 tests on PE100 pipe, which illustrate the effects of internal and external scoring on RCP resistance. Many test parameters are involved (including pressure, temperature, initiation conditions, propagation zone notch geometry and depth), and a full study would involve very extensive testing. However, the authors are able to draw sufficient conclusions to guide 
(a)

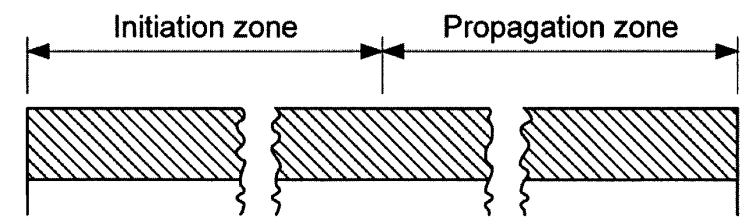

(b)

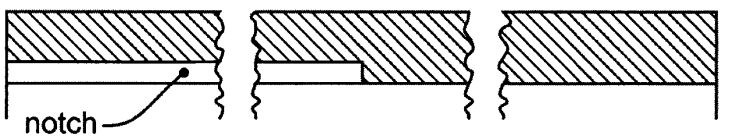

(c)

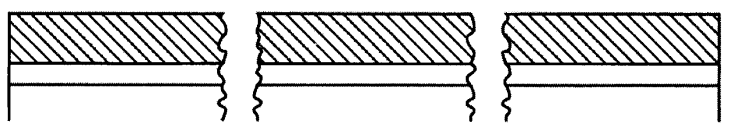

(d)

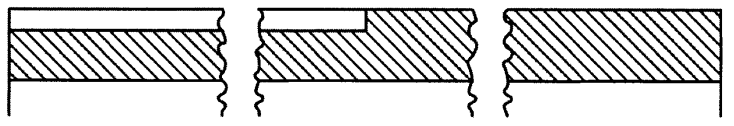

(e)

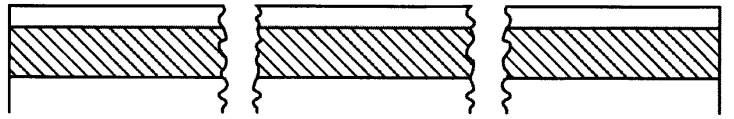

(f)
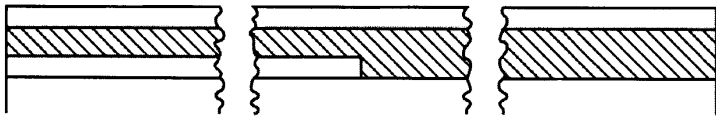

3 Schematic axial cross-sections through $\mathrm{S} 4$ pipe specimen walls, showing notch configurations tested. Ratio of notch depth to wall thickness is exaggerated

such a programme in future and to comment on the practical implications.

\section{Experimental}

The pipes used were extruded by Uponor Ltd from one batch of a typical commercial PE100. The pipe size was ' $125 \mathrm{SDR} 11$ ', i.e. $125 \mathrm{~mm}$ o.d., with an outside diameter $D$ to wall thickness ratio of 11 . Specimens were cut to the standard length of $7 D$ and both ends were deburred to facilitate sealing, but they were not prepared in any way other than by the introduction of sharp, axial surface notches. At room temperature, PE is easily scored by a razor blade, but its flexibility makes notch depth control difficult. To achieve maximum repeatability, each pipe was clamped in the stationary chuck of a lathe. A fresh razor blade was fitted into the end of a metal bar whose other end was attached to the tool post. The lathe lead-screw was then used to draw the blade through at constant speed, taking about $2 \mathrm{~min}$. For the present, relatively limited study, the nominal notch depth was kept constant at $1.5 \mathrm{~mm}$; this is the usual depth of an S4 test initiation notch. Each pipe to be tested below room temperature was then transferred to a conditioning unit and left there for at least $12 \mathrm{~h}$ before testing, and for at least three further hours after small $\left(2-3^{\circ} \mathrm{C}\right)$ temperature changes.

\section{Results}

The baseline performance of pipe without surface scores was first tested using the standard S4 'critical temperature' method (Annex A of ISO 13477). Because of the limited number of specimens available, $T_{\mathrm{c}}$ could not be determined with the accuracy of which the S4 method is capable. Specimens were conventionally notched for initiation (Fig. 3b). Figure 4 shows data from two test

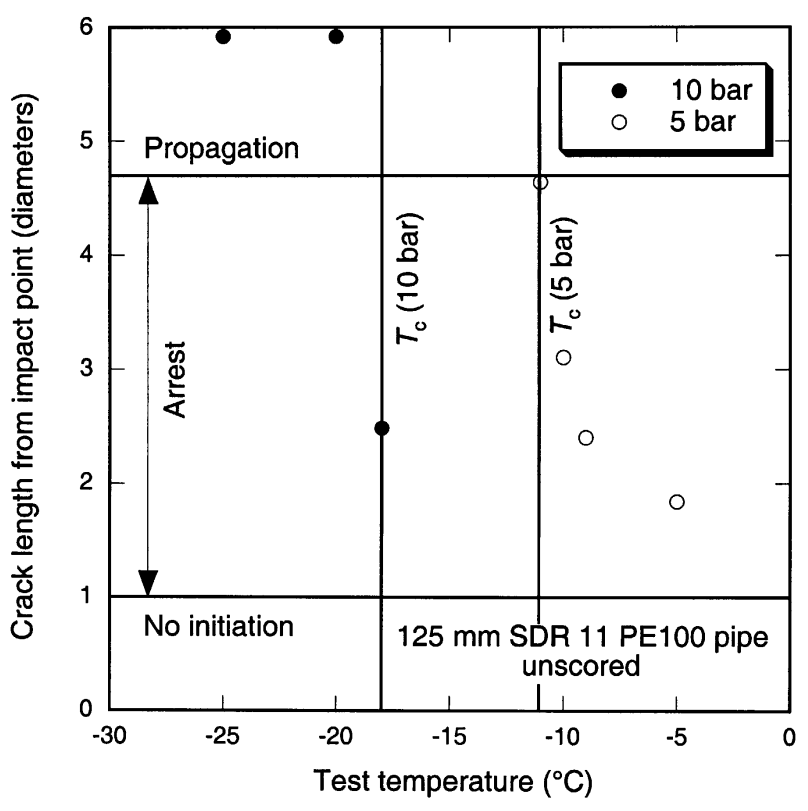

4 S4 test results from unnotched specimens

pressures, 5 and 10 bar. The effect of test pressure on $T_{\mathrm{c}}$ is a live issue within the current review of ISO 13477. Although Annex-A critical temperature testing is becoming more widely used than critical pressure testing, there is no agreed criterion for choosing the constant test pressure. The current de facto standard of 10 bar is probably too high: once the correlation factor referred to above is applied, it corresponds to a full-scale test pressure of more than 30 bar.

Figure 4 indicates a 10-bar transition temperature of $-18^{\circ} \mathrm{C}$. There is a $+0,-2$ bar uncertainty, but since the crack arrested at $-18^{\circ} \mathrm{C}$ with a length of 1.84 diameters - comfortably more than the initiation criterion of 1.0 and less than the propagation criterion of $4.7-T_{\mathrm{c}}$ is probably about $-19^{\circ} \mathrm{C}$. The 5 bar critical temperature shows a significantly higher value of $-10^{\circ} \mathrm{C}$. Evidently, critical temperature does depend on pressure, and it does so in an anomalous sense given that critical pressure decreases monotonically with temperature. ${ }^{7}$ The explanation perhaps lies in the mechanics of the test method, by which high pressure can 'overdrive' and blunt a crack without allowing it to run faster. On the other hand, the 5 bar results hint at another potential problem: the transition is noticeably softer. Since the critical pressure tends to settle to a lower shelf with decreasing temperature, RCP behaviour tends to become progressively less dependent on pressure. Testing at a fixed, low pressure leads to a less 'well conditioned' system and tends to result in greater scatter.

\section{Effect of internal notch}

To investigate the influence of the plane stress region at the internal pipe surface, an internal notch of $1.5 \mathrm{~mm}$ was cut all the way along the bore of some specimens (Fig. 3c).

Results are shown in Fig. 5. Again, it was not possible to test enough specimens to pinpoint the critical temperature. According to ISO13477, $T_{\mathrm{c}}$ is defined from the lowest temperature arrest result which lies above the highest temperature propagation result, and in this case these points are separated by an uncertainty of $5^{\circ} \mathrm{C}$. 


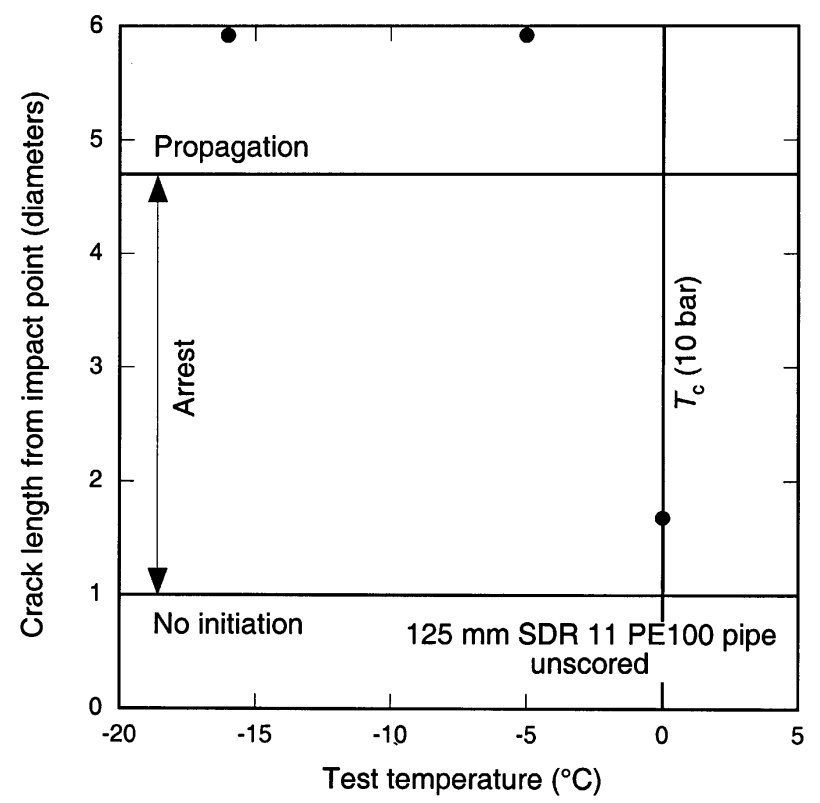

5 S4 test results from internally notched specimens

However, the upward shift in critical temperature caused by the surface notch is unmistakeable.

In full scale tests, RCP usually generates a sinusoidal crack path. In S4 tests the crack path is usually straighter, but truly straight crack paths are normally associated with highly brittle fracture and with high speed propagation at low temperatures. Not surprisingly, in most tests on specimens at moderately low temperature but with internal notches, the crack followed the straight path of the notch. The fracture surface was brittle in appearance, with no shear lip at the notch root. The crack ran the whole length of the pipe specimen, but the fracture surface did record hesitations in propagation at which large shear zones were visible on the outside pipe surface; these crack arrest marks will be discussed later.

\section{Effect of external notch}

Two sets of experiments were carried out on pipe specimens with artificial external scores: one set without and one set with a conventional internal notch in the initiation section.

\section{Without initiation notch}

The intention of these tests was to investigate the external notch effect in isolation. Pipe specimens were prepared with $3 \mathrm{~mm}$ deep external notches extending either along the initiation section (Fig. $3 d$ ) or along the whole specimen (Fig. 3e). It was not possible to achieve valid initiation in any of these specimens above $-25^{\circ} \mathrm{C}$, so that no crack could propagate in the notched region. Below $-27^{\circ} \mathrm{C}$, however, this pipe material shows little ability anyway to develop the tough plane stress regions which normally effect crack arrest. Thus although the crack did then propagate, it seemed to do so independently of the notch, following a different path. Clearly, a short external notch, unlike the usual internal starter notch, does not provide a ready initiation point for RCP.

In terms of a standard S4 procedure, these are invalid tests. A constant temperature, critical pressure procedure normally begins with tests on unpressurised specimens, in order to determine impact parameters (notch depth and gas gun pressure) to generate a crack one diameter in length. For a critical temperature series this procedure is not defined. However, if initiation conditions are invalid (as they are here) then the fact is evident from the length of cracks at temperatures exceeding $T_{\mathrm{c}}$. After increasing the initiator gas gun pressure from 3.5 to 5 bar to increase the impact speed, another initiation test was carried out; again, no crack initiation was observed. Thus all the initiation tests were invalid, but their result was conclusive: an external notch does not significantly reduce the resistance of a pipe to $\mathrm{RCP}$ initiation.

Only at very low temperatures (e.g. $\left.-22^{\circ} \mathrm{C}\right)$ did the crack run if initiated. Even then, in some cases the crack even ran parallel to but alongside the axial notch. It can be concluded that if the pipe is externally notched but has no internal starter notch, the crack either will not start at all or, if the pipe is brittle enough, may run all the way along the pipe in its own random path as if ignoring the presence of the external notch.

\section{With initiation notch}

Use of an internal initiation notch, aligned with the external propagation score (Fig. 3f), restored a more conventional response to $\mathrm{S} 4$ testing.

Again, the test series was short. However, some specimens were initially used to explore the effect of external notch depth. When testing water-pressurised pipes with internal notches, Greenshields ${ }^{10}$ reported the existence of a critical notch depth below which RCP could not be sustained at any pressure: testing the same size of pipe (125 mm SDR 11), he found this to be $1.2 \mathrm{~mm}$. In the present series of tests at 10 bar on externally notched pipes, cracks arrested at intermediate lengths in specimens with 1.5 and $2.5 \mathrm{~mm}$ notch depth, but with a $3 \mathrm{~mm}$ deep notch, RCP was sustained throughout the propagation section. The external critical notch depth thus proves to be about $3 \mathrm{~mm}$, which for this pipe is $25 \%$ of the full wall thickness. At the same notch depth, RCP was sustained and the critical temperature was between 0 and $-5^{\circ} \mathrm{C}$; the RCP fracture surface at $-5^{\circ} \mathrm{C}$ was very brittle.

\section{Discussion}

The tests reported here were essentially exploratory in nature, and a more extensive and systematically planned programme is certainly needed. Although the results are generally consistent, they are in some ways surprising. The anomalous effect of pressure on critical temperature is the most disturbing of these results, but it is one of which laboratories using the test were aware. It is to be separately addressed by a change in the $\mathrm{S} 4$ standard to exclude testing above 6 bar, but the present programme was planned and conducted within a tradition which has generated much data at 10 bar.

A sharp, internal notch significantly increases the critical temperature for RCP in a polyethylene pipe. However, these authors have consistently observed that the effect of internal surface notching on an S4 specimen initiation region is temporary. Only if the specimen is rapidly cooled within 1 day or so (depending on material) after notching does the notch guarantee RCP 


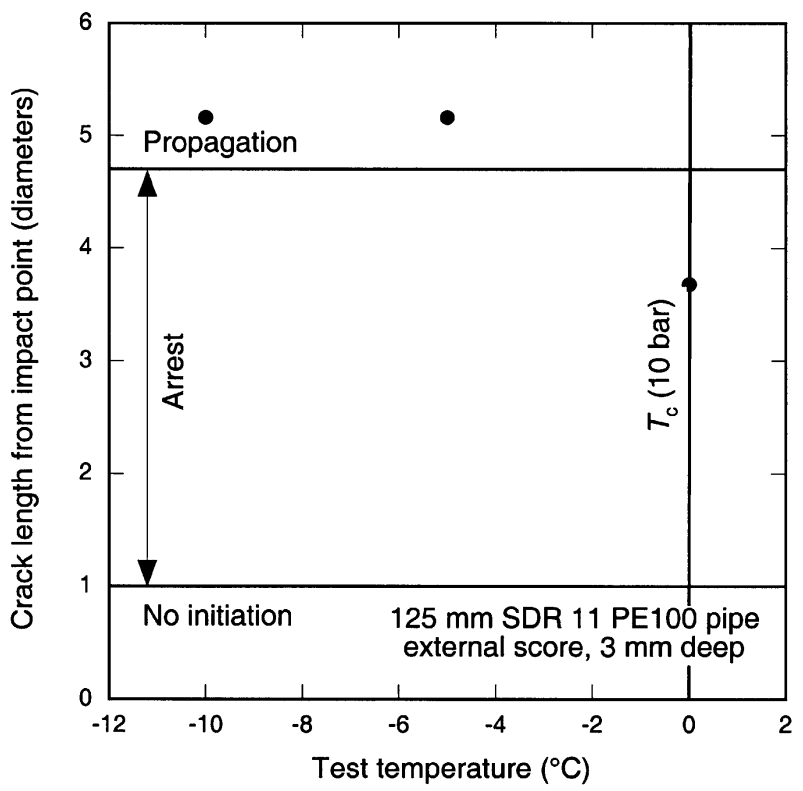

6 S4 test results from externally notched specimens with initiation notches

initiation after conditioning. This is believed to be a result of viscoelastic notch blunting under the tensile, circumferential, residual stress at the internal surface of the pipe.

An external initial notch did not facilitate crack initiation in the same way as an internal notch. Nevertheless, a very deep notch was, again, able to increase the critical temperature to approach $0^{\circ} \mathrm{C}$. Remarkably, however, a crack propagating above the 'unnotched' critical temperature due to the presence of an external notch was able to do so along a path which lay mostly outside and alongside the notch. It might be that when the crack crosses the notch it accelerates, but the external notch cannot initiate or control crack propagation in the same way as an internal notch, which usually exerts a powerful guiding effect on crack propagation. It is important to point out that installation procedures often impose their own limits on external scoring: for UK fuelgas networks, for example, scores deeper than $10 \%$ of the pipe wall thickness are unacceptable.

There could be a number of factors at work in defining this asymmetry. As mentioned above, the material of the inner surface is usually different, especially in PE100 resins whose structural complexity makes their fracture resistance rather sensitive to cooling rate. ${ }^{11}$ However, two mechanics factors may be more relevant. First, post-extrusion cooling of the pipe from the outside surface freezes in tensile stress at the bore. Thus a driving force for crack extension exists even if there is no pressure, since strain energy can be relieved by the wall buckling inwards at the crack plane; this driving force can add to that due to pressure. Secondly, as an axial crack front cuts through any section of pipe, the wall does not simply relax circumferentially: computer simulations ${ }^{12}$ confirm that if the pipe wall is allowed to flare outwards behind the crack front under gas pressure, then a buckling effect dimples the wall inwards around the crack tip itself (Fig. 8). It is true that flaring is restricted in the $\mathrm{S} 4$ test by the containment cage rings shown in Fig. 2; nevertheless, this may well be a significant effect.
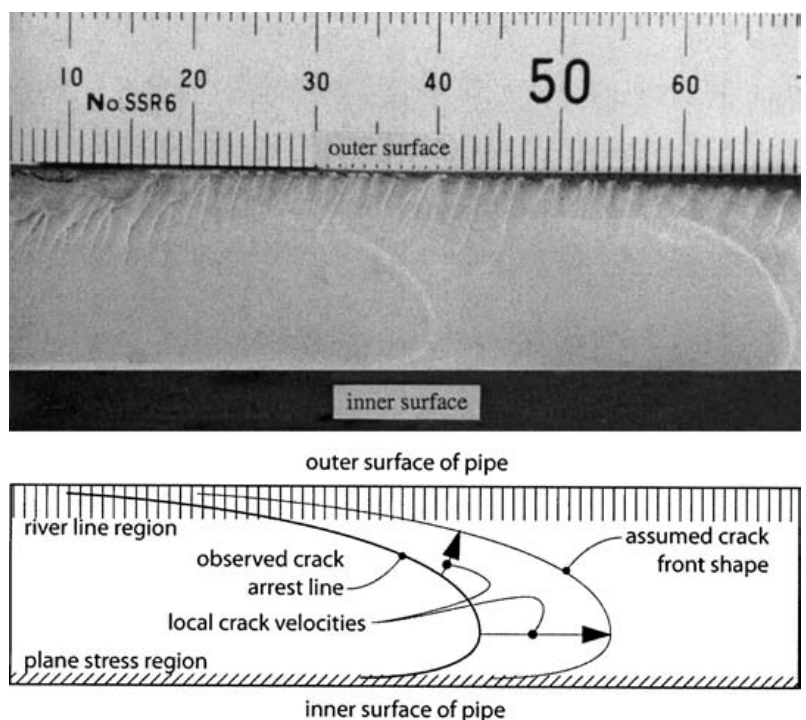

7 Pipe fracture surface from un-notched specimen showing typical arrest mark

It is probably because of this inward buckling at the crack tip that an internal notch so effectively guides the crack. This effect is seen in the Double Torsion geometry (Fig. 9), whose crack driving mode both of the effects above resemble. However, it is not easy to explain or model the effect precisely, since the criteria which determine crack path are not fully understood even in plates under purely two-dimensional, in-plane stress. In a Double Torsion specimen, ${ }^{13}$ the crack must usually be guided with a side-groove on the tension surface (the underside in Fig. 9) because a straight, central path along the axis of symmetry is otherwise only marginally stable. This lower side-groove is usually $\mathrm{V}$-shaped, but it seems to provide enough constraint to suppress plane stress deformation and its guiding effect proves to be even greater than the razor scores tested in this work.

However, the side-groove in a DT specimen does not essentially change the overall shape of the crack, which is a highly asymmetrical parabola (Fig. 9). When a brittle crack front propagating through PE arrests whether temporarily (before re-initiating) or permanently - it leaves a stress-whitened trace which is assumed to represent the crack front shape. These arrest marks are seen both in pipe, as shown in Fig. 6 and reported elsewhere ${ }^{8-10}$ and in DT specimens, ${ }^{13}$ and the crack front shapes are remarkably similar. Within the

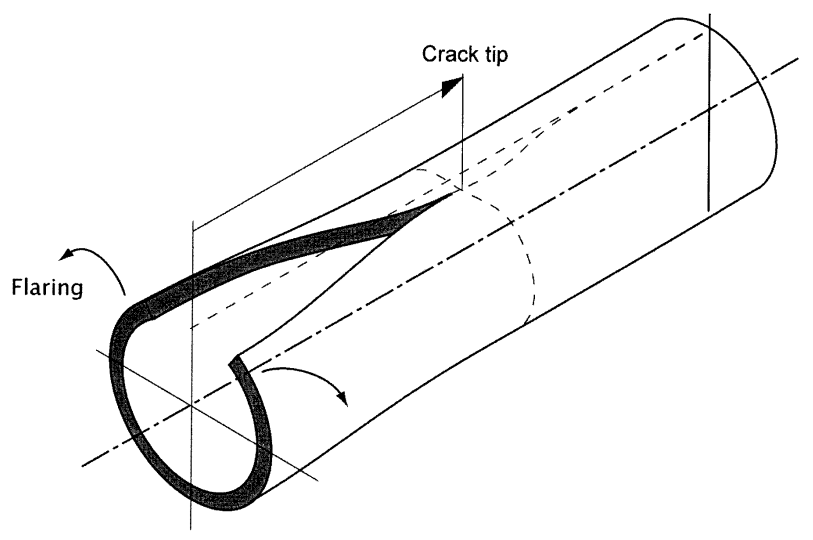

8 Flaring and dimpling of pipe wall during RCP 


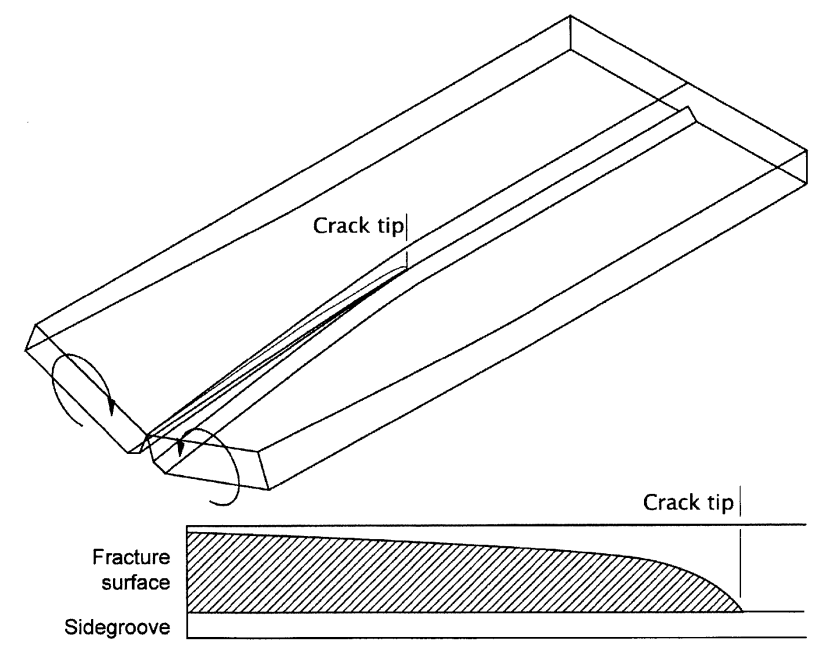

9 Schematic diagram of double torsion test and crack front shape

inner third of the wall section, the crack front appears to advance in a direction almost parallel to the axis ${ }^{6}$ except, owing to plane stress effects, immediately adjacent to the inner surface. Over the remaining surface, the crack front propagates mainly towards the outer edge.

In both specimen types, this shape results from the combined effect of simple circumferential opening arising from in-plane stretching, and out-of-plane bending. Tests on pipe subjected to dual surface cooling, which induces a symmetrical residual stress field, do show a straighter and more symmetrical crack front. ${ }^{14}$ This suggests that the residual stresses generated during pipe extrusion exert at least as much influence as the buckling generated by flaring, at least in the S4 test where flaring is deliberately reduced.

The present tests showed that when an external notch is present, the crack will still lead at the inner surface and propagate towards the outer surface, which is then in compression. Because the crack tends to follow a sinusoidal path, if an external notch is straight - and does not therefore prescribe the most probable path the crack only occasionally follows it. Only while the crack does so is the resistance of the material to it decreased, although it is possible that the effect may persist temporarily as a result of crack acceleration.

\section{Conclusions}

Sharp surface notches have a detrimental effect on the resistance of PE100 pipes to rapid crack propagation
(RCP), by suppressing the plane stress component of resistance to it - which, in this material, dominates the plane strain component. However, significant effects are seen only under unrealistically extreme conditions. An internal axial score can reduce resistance to initiation and (within its length) to propagation of a rapid crack. However, such scores are unlikely to be inflicted and they appear to self-blunt within days under a normal residual stress distribution. External axial scores affect RCP resistance only if they are so deep that normal service could not be expected anyway. Further research is needed to confirm these results at the lower S4 test pressures now used, to explore more thoroughly the effect of notch depth in different materials, and to quantify the self-blunting phenomenon under both residual and pressure-generated stress.

\section{Acknowledgements}

The authors wish to express their gratitude to Uponor Ltd who provided full and generous support for Luisa Moreno's PhD programme, including the work reported here.

\section{References}

1. R. Shannon and A. Wells: Int. J. Fracture, 1974, 10, 471-486.

2. J. M. Greig, P. S. Leevers and P. Yayla: Eng. Fracture Mech., 1992, 42, 663-673.

3. P. Vincent: Polymer, 1974, 15, 111.

4. P. S. Leevers: Int. J. Fracture, 1995, 73, 109-127.

5. M. A. Wheel and P. S. Leevers: Int. J. Fracture, 1993, 61, 349 359.

6. P. Yayla: 'Rapid crack propagation in polyethylene gas pipes', $\mathrm{PhD}$ thesis, University of London, London, UK, 1991.

7. R. E. Morgan: 'Ductile-brittle transitions in pipe grade polyethylene', PhD thesis, University of London, London, UK, 1994.

8. S. Youd: PhD thesis, Manchester Polytechnic, Manchester, UK, 1992.

9. L. Moreno and P. S. Leevers: 'Impact fracture toughness of polyethylene/polypropylene multilayers', Polym. Eng. Sci., to be published.

10. C. Greenshields: 'Fast brittle fracture of water/air pressurised plastic pipes', $\mathrm{PhD}$ thesis, University of London, London, UK, 1995.

11. S. J. K. Ritchie, P. Davis and P. S. Leevers: Polymer, 1998, 39, 6657-6663.

12. A. Ivankovic, V. Tropsa, H. Jasak and P. S. Leevers: in 'ECF $14-$ Fracture mechanics beyond 2000,' (ed. A. Neimitz et al.), Vol. 2, 21-28; 2002, Sheffield, EMAS Publishing.

13. P. S. Leevers and J. G. Williams: J. Mater. Sci., 1985, 20, 7784.

14. C. J. Greenshields, P. Davis and P. S. Leevers: Plast. Rubber Compos., 1997, 26, 404411. 


\section{Authors Queries}

Journal: Plastics, Rubbers and Composites

Paper: 2078

Title: Effect of axial surface scores on rapid crack propagation in polyethylene pipe

Dear Author

During the preparation of your manuscript for publication, the questions listed below have arisen. Please attend to these matters and return this form with your proof. Many thanks for your assistance

\begin{tabular}{|l|l|l|}
\hline $\begin{array}{l}\text { Query } \\
\text { Reference }\end{array}$ & Query & Remarks \\
\hline 1 & Author: Please supply keywords. & \\
\hline 2 & $\begin{array}{l}\text { Author: First mention of Figure 6 } \\
\text { and out of sequence. Please } \\
\text { insert a citation for this figure in } \\
\text { sequence. Figure 7 not men- } \\
\text { tioned at all. Please insert a } \\
\text { citation for Fig. 7 in sequence. }\end{array}$ & \\
\hline 3 & $\begin{array}{l}\text { Author: Any further details for } \\
\text { Ref. 9? }\end{array}$ & \\
\hline
\end{tabular}

